März 2014 zusammengeführt zu haben (Friedrich-Ebert-Stiftung, 2014). In großer Einigkeit plädierten Sozialpartner und Parlamentarier der Großen Koalition für ein neues Forschungs- und Aktionsprogramm zur Neugestaltung der Arbeit.

Die Entwicklung fand ihren vorläufigen Abschluss auf der BMBF-Tagung „Dienstleistungen in einer digitalen Welt“ im Mai 2014, in der das BMBF ein neues Arbeitsforschungsprogramm zusagte, das in Kooperation mit den Sozialpartnern erstellt werden soll.

Es ist nicht klar, was die Zukunft bringen wird, aber klar ist, dass die Anstrengungen von Wissenschaft und Wirtschaft, von Sozialpartnern und vielen anderen eine Basis gelegt haben, Arbeitswissenschaft und Arbeitsforschung voranzutreiben. Arbeitsforschung ist wieder da.
Tun wir alles, um sie am Leben zu halten.

\section{Literatur}

Barthel, Klaus: Humanisierung der Arbeit braucht Forschung, WISO direkt Mai 2013, Friedrich-Ebert Stiftung, Bonn (2013)

BMBF, ; BMA, : Forschung zur Humanisierung des Arbeitslebens - Dokumentation 1987, Eigenverlag, Bonn (1987)

Bsirske, Frank: Dienstleistungspolitik für Gute Arbeit und Gute Dienstleistungen, Rede auf der Tagung Dienstleistungsforschung - Dienstleistungspolitik der Hans-Böckler-Stiftung und ver. di am 24. April 2013; http://www.boeckler.de/pdf/v_2013_04_24_25_bsirske.pdf (2013)

Friedrich-Ebert-Stiftung: http://www.fes.de/ wiso/content/veras/v_arbeit_betrieb_politik. php\#2013 (2014)

Negt, Oskar: Arbeit und menschliche Würde, Aus Politik und Zeitgeschichte, Beilage zur Wochenzeitung DAS PARLAMENT, Nr. 15, S. 3-5 (2011)
Schweres, Manfred: Editorial: Für eine neue $\mathrm{Hu}-$ manisierungsoffensive, Zeitschrift für Arbeitswissenschaft, Bd. 63, S. 89 (2009)

Schweres, Manfred: Editorial: Für eine neue, nachhaltigere, innovations- und produktivitätsförderliche Humanisierungsoffensive, Zeitschrift für Arbeitswissenschaft, 67. Jahrgang, S.1-3 (2013)

Ulich, Eberhard; Zink, Klaus J., Kubek, Vanessa: Das Menschenbild in Arbeitswissenschaft und Betriebswirtschaftslehre, Zeitschrift für Arbeitswissenschaft, 67. Jahrgang, S. 15-22 (2013)

Zink, Klaus J.: Die Zukunft der Arbeit erforschen. Ein Memorandum der Gesellschaft für Arbeitswissenschaft e.V. zum Strukturwandel der Arbeit., Dortmund (2000)

\section{Anschrift des Verfassers}

Gerhard Ernst

Kirchstraße 36

56316 Raubach

E-Mail: kirchstrasse36@gmail.com

\title{
Herausforderungen moderner Arbeitsweltgestaltung - Themen für eine innovative Arbeitsforschung
}

Die deutsche Wirtschaft steht kontinuierlich vor der Herausforderung, die Wettbewerbsfähigkeit der Unternehmen durch innovative und produktive Prozesse und Produkte nachhaltig zu stärken. Dafür braucht sie leistungsfähige, qualifizierte und motivierte Beschäftigte. Die Gestaltung neuer Arbeitswelten nimmt daher erheblich an Bedeutung zu. Wesentliche Aufgaben - wie sie derzeit in zahlreichen Unternehmen beobachtet werden - sind:

\section{Sicherung der körperlichen und geisti-} gen Leistungsfähigkeit

- Alternsgerechte Arbeitsgestaltung für Produktions-, Büro- und sonstigen Arbeitssystemen

- Belastungsoptimierende Rotationsmodelle

- Psychische Belastung am Arbeitsplatz

- Lebenslanges Lernen zur Stabilisierung der kognitiven Leistungsfähigkeit

2. Gestaltung von flexiblen, lebensphasenorientierten Arbeitszeitsystemen

- Arbeitszeit als entscheidendes Element zur Entspannung nach den beruflichen Anspannungsphasen derzeit viele Piloten für ergonomische, flexible und lebensphasenorientierte Arbeitszeitsysteme, eine wissenschaftliche Absicherung existiert nicht

\author{
- z. B. fehlen arbeitswissenschaftlich \\ gesicherte Forschungsstudien im Be- \\ reich der flexiblen Arbeitszeiten
}

3. Digitalisierung und Organisation der Arbeit in modernen Produktionssystemen - Industrie 4.0 gegenwärtig vorwiegend unter technologischen Gesichtspunkten diskutiert

- Arbeit und Arbeitsorganisation in der Industrie 4.0 völlig unterbelichtet - die arbeitspolitische Gestaltung beeinflusst wesentlich den volks- und betriebswirtschaftlichen Erfolg der Industrie 4.0

- Fragestellungen sind vielfältig: Qualifizierung, Entgelt, Arbeitszeit, Arbeitsschutz, Arbeitsorganisation, Führung

4. Design von heterogenen Beschäftigungsformen und vielschichtigen Karrierepfaden

- präventiver Ansatz durch Personalentwicklung: bedarfsorientiert und altersübergreifend

- Lern- und Veränderungsfähigkeit erhalten durch lebenslanges Lernen; wie funktioniert die Qualifizierung „lernentwöhnter“ (älterer) Mitarbeiter

- Laufbahngestaltung bei älter werdenden Belegschaften, Erforschung neuer Karrierepfade und Führungsmodelle im Unternehmen.
Bewertung der Eschborner Thesen zur Arbeitsforschung

Die Bedeutung und Notwendigkeit einer starken Arbeitsforschung ist grundsätzlich richtig - in Nuancen jedoch arbeitgeberfern.

\section{Umfassende Arbeitsforschung relevant} moderne Arbeitswelten (z. B. neue Technologien, Flexibilisierungsansätze)

lückenhafter Kenntnisstand in manchen Themen (z. B. arbeitsbezogene psychische Belastung, lebenslanges Lernen)

Kritikpunkte an der Formulierung, u. a. Ausschließliche Orientierung an $\mathrm{Hu}$ manität, womit nur eine Seite betrachtet wird; Wirtschaftlichkeit muss als weitere Maxime gelten.

Aktuelle Arbeitswelt wird zu negativ dargestellt („,bad jobs“, „prekär“...)

Teilzeitarbeit wird arbeitgeberseitig nicht als prekäre Beschäftigung verstanden, wie es in den Thesen subsumiert wird. Teilzeitarbeit ist sehr oft von den Beschäftigten erwünscht, kommt diesen entgegen und ist keinesfalls prekär.

- Zunahme AU-Tage ausschließlich aufgrund der psychischen Belastung in der Arbeitswelt keineswegs richtig. 